\title{
Future sensitivity on unitarity, light-sterile neutrinos and neutrino magnetic moment from low-energy experiments
}

\author{
O. G. Miranda, ${ }^{a}$ D. K. Papoulias, ${ }^{b}$ O. Sanders, ${ }^{a, *}$ M. Tórtola ${ }^{c, d}$ and J. W. F. Valle ${ }^{d}$ \\ ${ }^{a}$ Departamento de Física, Centro de Invesgicación y de Estudios Avanzados del IPN, \\ Apartado Postal 14-750 07000 Mexico, Distrito Federal, Mexico \\ ${ }^{b}$ Division of Theoretical Physics, University of Ioannina, \\ GR 45110 Ioannina, Greece \\ ${ }^{c}$ Departament de Física Teórica, Universitat de Valencia, \\ Burjassot 46100, Spain \\ ${ }^{d}$ AHEP Group, Institut de Física Corpuscular - CSIC/Universitat de Valencia, \\ C/ Catedrático José Beltrán, 2E-46980 Paterna, Spain \\ E-mail: omr@fis.cinvestav.mx, dimpap@cc.uoi.gr, osanders@fis.cinvestav.mx, \\ mariam@ific.uv.es, valle@ific.uv.es
}

\begin{abstract}
We study the future sensitivities to a non-unitarity neutrino mixing matrix for various proposed short-baseline coherent elastic neutrino-nucleus scattering (CEvNS) experiments. We also identify the best configuration for measuring the oscillation parameters on the $(3+1)$ scheme for light sterile neutrinos and find the estimated sensitivity for their parameters. Finally, we study the conversion to massive sterile neutrinos (in the keV-MeV energy mass) through transition magnetic moments and find the sensitivities for actual COHERENT results as well as future experiments of CEvNS and elastic neutrino-electron scattering (EvES) with a proposed Cr-51 neutrino source experimental setup.
\end{abstract}

\footnotetext{
*** Particles and Nuclei International Conference - PANIC2021 ***

*** 5 - 10 September, $2021 * * *$

*** Online ***
}

\footnotetext{
${ }^{*}$ Speaker
} 


\section{Introduction}

We take into account three different scenarios:

- A) The presence of extra singlet heavy neutral leptons that mediate light-neutrino mass generation.

- B) The case in which the new sterile state is light enough to participate in oscillations.

- C) Neutrino magnetic properties in the presence of electroweak singlet neutrinos which allows the sterile dipole portal.

For the analysis of A) and B) we focus on three future experiments aiming to deploy large liquid argon detectors in order to measure CEvNS using a $\pi-D A R$ neutrino source. The summary of these considerations are shown in Table 1.

\begin{tabular}{|c|c|c|c|}
\hline & CENNS & CCM & ESS \\
\hline mass & $610 \mathrm{~kg}$ & 7 ton & $10 \mathrm{~kg}(1$ ton $)$ \\
threshold $\left(\mathrm{keV}_{n r}\right)$ & 20 & 1 & $0.1(20)$ \\
$N_{\text {POT }}\left(10^{23} / y r\right)$ & 1.5 & 0.177 & 2.8 \\
$\mathrm{r}$ & 0.08 & 0.425 & 0.3 \\
baseline $(\mathrm{m})$ & 28.4 & 20 & 20 \\
\hline
\end{tabular}

Table 1: Experimental configurations [1].

For C) we consider present and future CEvNS and EvES experiments. We also confirmed previous calculations regarding XENON1T which served as a calibration for our procedure in the study of TMM's. The free EvES cross section is weighted by a step function correction to take into account electron binding effects in each target material.

We are also motivated to explore the sterile dipole portal using a low-energy monochromatic $v_{e}{ }^{51} \mathrm{Cr}$ source through CEvNS and EvES processes. For this, we focus on a proposal for measuring CEvNS using a $5 \mathrm{MCi}^{51} \mathrm{Cr}$ source and different kg-scale detectors. Also, we examine the potential of a ${ }^{51} \mathrm{Cr}$-LXe detector through EvES measurements.

For the statistical analysis, in general we perform a $\chi^{2}$ calculation given by:

$$
\chi^{2}(S)=\sum_{i=1}^{n}\left(\frac{N_{i}-N_{i}^{n e w}(S)[1+a]}{\sigma_{i}^{\text {stat }}}\right)^{2}+\left(\frac{a}{\sigma_{\text {sys }}}\right)^{2} .
$$

\section{Results}

For A) we give a first estimate on the study of probing the unitarity-violating parameters for the future LAr detectors. First we only vary one parameter at a time, marginalizing over the other two, and imposing constraints from the triangular inequalities that they must obey. From here, one sees that CEvNS experiments cannot compete with current bounds, but they might become in the future competitive with the current constraints from oscillation searches. This is the case of 

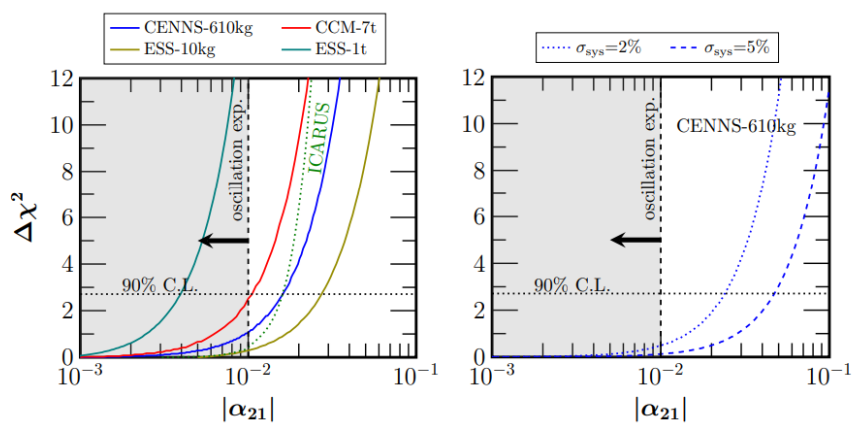

Figure 1: Sensitivity on the non-diagonal parameter $\left|\alpha_{21}\right|[1]$.

the next-generation of ESS. The right panel of Fig. 1 shows the projected sensitivities taking into account different systematic uncertainties.

It is also possible to perform a combined $\chi^{2}$ analysis varying two of the NU parameters, and marginalizing over the third one. We show our results for this on Fig. 2, here, the dark-shaded areas located to the right of each line are allowed at $90 \%$ C.L. by each experiment. We can see that CENNS and CCM may have the potential to probe part of the allowed parameter space. And as before the most promising experiment is the ESS in its future configuration.

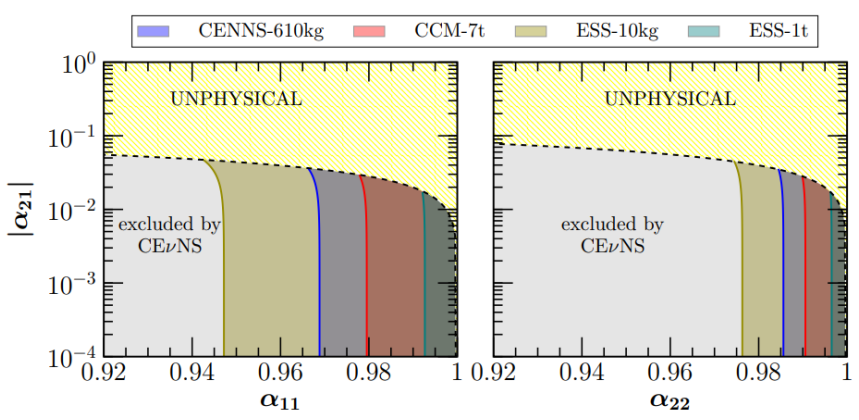

Figure 2: Allowed regions at 90\% C.L..The yellow region is unphysical due to triangular inequalities [1].

For the case of sterile neutrino oscillations we can explore how the parameter space can be probed by CEvNS measurements. Fig. 3 shows the sensitivity at $90 \%$ C.L. for each experimental proposal. From the results we arrive at the same general conclusion as before.

Finally, assuming the neutrino magnetic moment as an effective parameter we perform a sensitivity analysis for all the experiments discussed above. We show them at 90\% C.L. in Fig. 4.

\section{Conclusions}

We analyzed the potential of future CEvNS experiments in probing physics associated to the presence of heavy isosinglets neutrinos, light sterile neutrinos and to transition into a sterile massive neutrino state through a dipole moment interaction. We finally showed that future short-baseline experiments may become competitive and will test regions of interest for each of the different scenarios. 


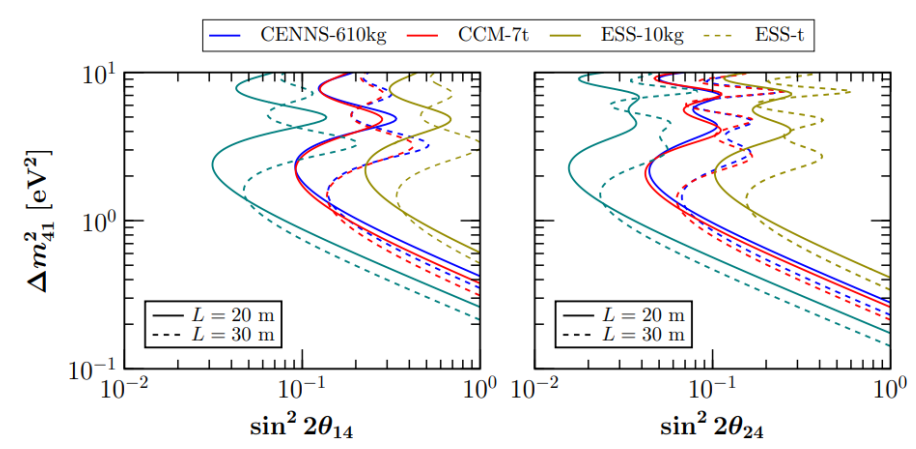

Figure 3: $90 \%$ C.L. sensitivity curves in the $\sin ^{2} 2 \theta_{i 4}-\Delta m_{41}^{2}$ plane for different baselines and experiments [1].

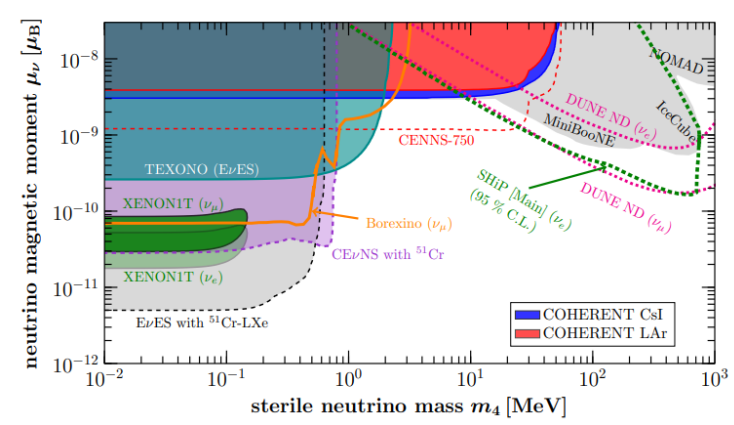

Figure 4: Sensitivity of CEvNS and EvES experiments for the effective sterile neutrino transition magnetic moment [2].

\section{Acknowledgments}

Work supported by the Spanish grant PID2020-113775GB-I00 (AEI / 10.13039/501100011033), PROMETEO/ 2018/165 (Generalitat Valenciana), by Fundacão para a Ciência e a Tecnologia (FCT, Portugal) through grant 19 CERN/FIS-PAR/0004/2019 and by CONACYT-Mexico under grant A1-S-23238. O. G. M. has been supported by SNI (Sistema Nacional de Investigadores). The work of DKP is co-financed by Greece and the European Union (European Social Fund-ESF) through the Operational Programme "Human Resources Development, Education and Lifelong Learning" in the context of the project "Reinforcement of Postdoctoral Researchers - 2nd Cycle" (MIS-5033021), implemented by the State Scholarships Foundation (IKY).

\section{References}

[1] O. G. Miranda, D. K. Papoulias, O. Sanders, M. Tórtola and J. W. F. Valle, Phys. Rev. D 102, 113014 (2020) doi:10.1103/PhysRevD.102.113014 [arXiv:2008.02759 [hep-ph]].

[2] O. G. Miranda, D. K. Papoulias, O. Sanders, M. Tórtola and J. W. F. Valle, [arXiv:2109.09545 [hep-ph]]. 